\title{
Methoden zur Effizienzmessung in der deutschen Wasserversorgung - Eine Auswertung internationaler Studien
}

\author{
DEA; SFA; Strukturparameter; Kostentreiber; Regulierung
}

In der deutschen Wasserwirtschaft existieren große Unterschiede bei der Höhe der zu entrichtenden Wasserpreise. Die unterschiedlichen strukturellen Gegebenheiten können einen Teil dieser Unterschiede begründen. Ein weiterer Teil ist womöglich auf Effizienzunterschiede zwischen den Unternehmen zurückzuführen. Um diese unter Berücksichtigung der jeweiligen strukturellen Besonderheiten zu bestimmen, muss der Vergleich der Unternehmen mit Hilfe wissenschaftlicher Effizienzmessungsmethoden durchgeführt werden. In diesem Artikel werden ausgehend von internationalen Studien Empfehlungen für die Anwendung einer Effizienzmessung in der deutschen Wasserwirtschaft unterbreitet.

\section{Einführung}

Der allägliche Gebrauch von Trinkwasser ist in der heutigen Zeit nicht mehr wegzudenken. Trinkwasser ist lebensnotwendig und zudem schlecht substituierbar. Wegen vermeintlich überhöhter Wasserpreise geraten gegenwärtig die deutschen Wasserversorgungsunternehmen (WVU) zunehmend unter Druck. So wurde die Verfügung des Landeskartellamtes Hessen gegen die Enwag $\mathrm{GmbH}$ aus Wetzlar, die Wasserpreise um fast $30 \%$ zu senken, kürzlich vom Bundesgerichtshof weitestgehend bestätigt (BGH 2010). Dieses Urteil könnte das weitere staatliche Vorgehen beim Umgang mit Unternehmen der Wasserversorgung präjudizieren.

Es ist jedoch zu berücksichtigen, dass die WVU in den einzelnen Städten und Gemeinden sehr unterschiedliche und nicht beeinflussbare Voraussetzungen vorfinden. Diese haben große Auswirkungen auf die Kosten und somit auch auf die Wasserpreise (von Hirschhausen 2009, S. 150 f.). Um diese abweichenden Umstände angemessen bei einer Analyse der Wasserentgelte zu berücksichtigen und somit zu belastbaren Aussagen bezüglich der Kosteneinsparpotentiale zu gelangen, besteht Einigkeit darüber, dass der Effizienzvergleich zwischen den Unternehmen anhand fortgeschrittener wissenschaftlicher Effizienzanalysen erfolgen sollte. ${ }^{1}$ Ziel des Beitrags ist demzufolge, auf Basis der internationalen Studien Rückschlüsse zur Anwendung der Effizienzanalysen bei deutschen Wasserversorgern abzuleiten.

Dazu werden zunächst kurz die Methoden zur Effizienzmessung definiert und darauf aufbauend internationale Studien vorgestellt. Anhand dieser werden Rückschlüsse für den Einsatz der Me-

1 Es ist anzumerken, dass bilanzpolitische Maßnahmen und kalkulatorische Spielräume sich ebenfalls auf die Wasserpreise auswirken. Der Fokus dieses Beitrages liegt jedoch lediglich auf den strukturellen Gegebenheiten und deren Auswirkungen auf den berechneten Effizienzwert und damit auf den Wasserpreis. 
thoden zur Effizienzmessung in der deutschen Wasserwirtschaft abgeleitet. Auch aus dem Einsatz in der Anreizregulierung in der deutschen Strom- und Gasbranche können Erkenntnisse gewonnen werden, da es sich sowohl bei der Wasserversorgung als auch bei der Strom- und Gasversorgung um netzgebundene Branchen handelt. Abschließend werden Limitationen für besondere Situationen aufgezeigt und spezielle Handlungsempfehlungen gegeben.

\section{Methoden zur Effizienzmessung}

Deutschland weist im internationalen Vergleich eine sehr kleinteilige Struktur der Trinkwasserversorgung auf, da die Wasserversorgung Aufgabe der Kommunen ist. So existierten im Jahr 2007 in Deutschland 6.211 WVU, bzw. 75,5 WVU pro eine Million Einwohner (zum Vergleich: Niederlanden 4,4; England/Wales 0,7; Frankreich 0,13 (Oelmann 2005, S. 23)). Aufgrund der strukturellen Unterschiede der WVU sind weder einfache Kennzahlenvergleiche (z. B. Kosten pro versorgten Haushalt) noch analytische Kostenmodelle zweckmäßig. Aus wissenschaftlicher Sicht sind lediglich parametrische und nicht-parametrische Verfahren zur Ermittlung der Effizienz einsetzbar.

Die Data Envelopment Analysis (DEA) ist das bedeutendste nicht-parametrische Verfahren zur Effizienzmessung. Die Effizienz einzelner Unternehmen wird dabei im Verhältnis zu einer Gruppe von Vergleichsunternehmen gemessen. Dazu wird mittels der linearen Programmierung eine Effizienzgrenze bestimmt (Oelman u. a. 2009, S. 841). Der Vorteil dieser Methode liegt darin, dass die Gewichte der In- und Outputs endogen aus dem Optimierungsmodell abgeleitet werden (Dyckhoff/Allen 1999, S. 420). Mittels einer nachgelagerten Regressionsrechnung können auch verschiedene Strukturparameter in die Berechnung einfließen und berücksichtigen dadurch die spezifischen Umweltbedingungen der einzelnen WVU (Zschille/Walter/von Hirschhausen 2010, S. 207). Bei Abweichung von der Effizienzlinie kann jedoch nicht zwischen Ineffizienzen und Datenfehlern unterschieden werden, so dass die gesamte Abweichung als Ineffizienz ausgewiesen wird und diese daher ggf. höher ausfällt (Jensen u. a. 2008, S. 30).

Diesen Nachteil korrigiert die bekannteste parametrische Methode, die Stochastic Frontier Analysis (SFA). Es wird ein funktionaler Zusammenhang zwischen den In- und Outputs in Form einer Produktions- bzw. Kostenfunktion angenommen. Die Abweichungen der Unternehmen werden getrennt in nicht-negative Zufallsvariablen, die die Ineffizienzen der Unternehmen angeben, und einen symmetrisch verteilten Störterm, der Messfehler und zufällige Ereignisse (wie z. B. Unwetter) abbildet (von Hirschhausen/Walter/Zschille 2009, S. 171). Die SFA unterstellt jedoch implizit, dass alle zu vergleichenden Unternehmen mit der gleichen Technologie arbeiten, was bei ca. 6.200 deutschen WVU nicht realistisch ist (Kletzan/Url 2003, S. 389).

Andere parametrische Verfahren wie die Ordinary Least Squares (OLS), die Corrected Ordinary Least Squares (COLS) und die Modified Ordinary Least Squares (MOLS) sind nicht ohne Weiteres für einen Effizienzvergleich zu gebrauchen, da sie entweder nur eine Durchschnittsbetrachtung liefern, sehr stark von Ausreißern abhängen oder nicht zwischen Ineffizienzen und zufälligen Schocks unterscheiden können. 


\section{Darstellung der verwendeten Studien}

Diverse internationale Studien (vgl. Tabelle 1) haben sich bereits der Effizienzmessung in der Wasserwirtschaft angenommen. Anhand dieser internationalen Erfahrungen werden Rückschlüsse für eine Effizienzmessung in Deutschland gezogen. Dabei werden alle Phasen des Effizienzvergleichs in den folgenden Kapiteln anhand eines dreistufigen Vorgehens betrachtet.

\begin{tabular}{|c|c|c|c|}
\hline Autoren (Jahr) & Daten & Methode & Untersuchungsschwerpunkt \\
\hline Aubert/Reynaud (2005) & $\begin{array}{l}\text { Panal Daten von } 211 \text { WVU aus } \\
\text { Wisconsin aus } 1998-2000\end{array}$ & SFA mit Translog-Kostenfkt. & $\begin{array}{l}\text { Effekt der Regulierung auf die Ef- } \\
\text { fizienz }\end{array}$ \\
\hline Coelli/Walding (2005) & $\begin{array}{l}18 \text { größte WVU Australiens aus } \\
1995 / 6 \text { bis } 2002 / 3\end{array}$ & $\begin{array}{l}\text { DEA (CRS + VRS) und Malm- } \\
\text { quist TFP Index }\end{array}$ & $\begin{array}{l}\text { Effizienzwerte des Jahres 2002/3 } \\
\text { und Entwicklung seit 1995/6 }\end{array}$ \\
\hline Estache/Kouassi (2002) & $\begin{array}{l}\text { 21 Afrikanische WVU aus } \\
\text { 1995-1997 }\end{array}$ & $\begin{array}{l}\text { SFA mit Cobb-Douglas-Pro- } \\
\text { duktionsfkt. }\end{array}$ & $\begin{array}{l}\text { Effizienzwerte in Abhängigkeit } \\
\text { von der Unternehmensform und } \\
\text {-größe }\end{array}$ \\
\hline Estache/Rossi (2002) & $\begin{array}{l}\text { Daten von } 50 \text { WVU Asiens aus } \\
1995\end{array}$ & $\begin{array}{l}\text { SFA mit Cobb-Douglas-Kos- } \\
\text { tenfkt. }\end{array}$ & $\begin{array}{l}\text { Einfluss der Organisationsform auf } \\
\text { die Effizienz }\end{array}$ \\
\hline Filippini/Hrovatin/Zoric (2008) & $\begin{array}{l}\text { Panal Daten von } 52 \text { Sloweni- } \\
\text { schen WVU aus } 1997-2003\end{array}$ & $\begin{array}{l}\text { SFA in verschiedenen Ausfüh- } \\
\text { rungen }\end{array}$ & $\begin{array}{l}\text { Robustheit der Ergebnisse unter } \\
\text { verschiedenen Modellvarianten }\end{array}$ \\
\hline García-Sánchez (2006) & 24 spanische WVU & $\begin{array}{l}\text { Inputorient. DEA - CRS u. - } \\
\text { VRS }\end{array}$ & $\begin{array}{l}\text { Technische und Skalen-Ineffizien- } \\
\text { zen }\end{array}$ \\
\hline García-Valiñas/Muñiz (2007) & $\begin{array}{l}\text { Daten aus 1985-2000 von WVU } \\
\text { aus Gijon/Sevilla und Elche }\end{array}$ & Inputorientierte DEA & $\begin{array}{l}\text { Effizienz mit und ohne Berücksich- } \\
\text { tigung der nicht-kontrollierbaren } \\
\text { Variable "'Regenmenge"' }\end{array}$ \\
\hline Haug (2006) & 43 ostdeutsche WVU aus 2002 & $\begin{array}{l}\text { DEA und OLS-Schätzung einer } \\
\text { Cobb-Douglas-Produktionsfkt }\end{array}$ & $\begin{array}{l}\text { Skaleneffekte/konnten nicht nach- } \\
\text { gewiesen werden }\end{array}$ \\
\hline Haug (2007) & 43 ostdeutsche WVU aus 2002 & 2-stufige DEA & $\begin{array}{l}\text { Einfluss der Organisationsform auf } \\
\text { die Effizienz }\end{array}$ \\
\hline $\operatorname{Lin}(2005)$ & $\begin{array}{l}\text { Panel Daten von } 36 \text { WVU Perus } \\
\text { aus den Jahren 1996-2001 }\end{array}$ & $\begin{array}{l}\text { SFA mit Cobb-Douglas-Kos- } \\
\text { tenfkt und verschiedenen Effi- } \\
\text { zienztermverteilungen }\end{array}$ & $\begin{array}{l}\text { Einfluss der Qualität auf die Effi- } \\
\text { zienz }\end{array}$ \\
\hline $\begin{array}{l}\text { Kirkpatrick/Parker/Zhang } \\
\text { (2006) }\end{array}$ & $\begin{array}{l}76 \text { (SFA) bzw. } 66 \text { (DEA) Afri- } \\
\text { kanische WVU aus } 2000\end{array}$ & $\begin{array}{l}\text { Inputorientierte DEA VRS; } \\
\text { SFA }\end{array}$ & $\begin{array}{l}\text { Einfluss der Organisationsform auf } \\
\text { die Effizienz }\end{array}$ \\
\hline Kletzan/Url (2003) & 46 bzw. 96 WVU aus Österreich & DEA-CRS und DEA-VRS & $\begin{array}{l}\text { Kaum Effizienzunterschiede bei } \\
\text { den WVU }\end{array}$ \\
\hline Oelmann u. a. (2009) & $\begin{array}{l}\text { Daten aus Rödl \& Partner Da- } \\
\text { tenbank deutscher WVU }\end{array}$ & DEA/SFA & $\begin{array}{l}\text { Spezifische Rahmenbedingungen } \\
\text { haben großen Einfluss auf Kosten }\end{array}$ \\
\hline $\begin{array}{l}\text { Picazo-Tadeo/Sáez-Fernández/ } \\
\text { González-Gómez (2008) }\end{array}$ & $\begin{array}{l}38 \mathrm{WVU} \text { aus Andalusien/Daten } \\
\text { aus } 2001\end{array}$ & $\begin{array}{l}\text { Outputorient. DEA/mit u. ohne } \\
\text { Berücksichtigung der Qualität }\end{array}$ & $\begin{array}{l}\text { Berücksichtigung der Qualität er- } \\
\text { höht Effizienzwerte/Reihenfolge } \\
\text { ändert sich kaum }\end{array}$ \\
\hline $\begin{array}{l}\text { Picazo-Tadeo/González- } \\
\text { Gómez/Sáez-Fernández (2009) }\end{array}$ & $\begin{array}{l}34 \mathrm{WVU} \text { aus Andalusien/Daten } \\
\text { aus } 2001\end{array}$ & $\begin{array}{l}\text { 3-stufiger DEA-Ansatz mit } \\
\text { SFA in 2. Stufe }\end{array}$ & $\begin{array}{l}\text { Einfluss von Umwelteinflüssen auf } \\
\text { die Ergebnisse }\end{array}$ \\
\hline $\begin{array}{l}\text { Picazo-Tadeo/Sáez-Fernández/ } \\
\text { González-Gómez (2009) }\end{array}$ & $\begin{array}{l}34 \mathrm{WVU} \text { aus Andalusien/Daten } \\
\text { aus } 2001\end{array}$ & $\begin{array}{l}\text { 2-stufige DEA mit Inputslack- } \\
\text { Identifizierung }\end{array}$ & $\begin{array}{l}\text { Einfluss von Umwelteinflüssen auf } \\
\text { die Ergebnisse und Vorliegen von } \\
\text { Input-slack }\end{array}$ \\
\hline Renzetti/Dupont (2008) & $\begin{array}{l}64 \mathrm{WVU} \text { aus Ontario (Kanada) } \\
1996\end{array}$ & 3-stufige DEA/VRS & $\begin{array}{l}\text { Einfluss von Umwelteinflüssen auf } \\
\text { die Ergebnisse }\end{array}$ \\
\hline $\begin{array}{l}\text { Saal/Parker/Weyman-Jones } \\
\text { (2007) }\end{array}$ & $\begin{array}{l}\text { Daten der } 10 \text { Wasser- und Ab- } \\
\text { wasser-unternehmen aus UK } \\
\text { von 1985-2000 }\end{array}$ & $\begin{array}{l}\text { SFA mit einer Inputdistanz- } \\
\text { funktion }\end{array}$ & $\begin{array}{l}\text { Einfluss der Privatisierung auf die } \\
\text { Effizienz der Unternehmen }\end{array}$ \\
\hline Tupper/Resende (2004) & $\begin{array}{l}20 \text { Wasser- und Abwasserunter- } \\
\text { nehmen aus Brasilien von } \\
\text { 1996-2000 }\end{array}$ & $\begin{array}{l}\text { 3-stufige DEA-VRS/outputori- } \\
\text { entiert }\end{array}$ & $\begin{array}{l}\text { Einfluss von Umwelteinflüssen auf } \\
\text { die Ergebnisse/Umsetzung der Er- } \\
\text { gebnisse in eine Regulierung }\end{array}$ \\
\hline $\begin{array}{l}\text { Zschille/Walter/von Hirschhau- } \\
\text { sen (2009) }\end{array}$ & $\begin{array}{l}373 \text { deutsche WVU aus der Was- } \\
\text { serstatistik } 2006\end{array}$ & $\begin{array}{l}\text { 3-stufige DEA-VRS/inputori- } \\
\text { entiert }\end{array}$ & $\begin{array}{l}\text { Vor allem kleine WVU besitzen } \\
\text { große Ineffizienzen }\end{array}$ \\
\hline
\end{tabular}

Tabelle 1: Übersicht der verwendeten Studien

Quelle: Eigene Darstellung 
Bei der Auswahl der Studien wurde Wert auf Aktualität gelegt. Keine der Studien wurde vor 2002 veröffentlicht. Ferner beziehen sie Effizienzvergleiche aus allen Kontinenten ein. Die Mehrheit der Studien vergleicht Wasserversorger innerhalb eines Landes oder einer Region, drei Studien vergleichen WVU in unterschiedlichen Ländern eines Kontinentes. Zwölf Studien verwenden die DEA, sechs die SFA und zwei sowohl die DEA als auch die SFA. Durch die Auswahl der Studien werden alle für Deutschland relevanten Untersuchungsschwerpunkte einbezogen, so dass sich aus den daraus abzuleitenden Rückschlüssen ein umfassendes Bild ergibt.

\section{Vor dem Effizienzvergleich}

\section{Datenerhebung}

Die Grundlage für jeglichen Vergleich zwischen Unternehmen ist eine einheitlich erhobene Datenbasis. In den zu untersuchenden Studien kommen verschiedene Verfahren zur Datengewinnung zum Einsatz. Einige der Studien erheben die Daten mittels eines Fragebogens, der an die einzelnen WVU verschickt wurde. Jedoch ist die Bereitschaft der Unternehmen, an einer solchen Untersuchung teilzunehmen, nicht besonders groß. So wurden teilweise Rücklaufquoten von nur 15,64\% (Haug 2006) erreicht. Auch andere Autoren erzielen mit 21,24\% (Garcia-Sanchz 2006) oder 40,9\% (Kletzan/Url 2003) keine zufriedenstellenden Rücklaufquoten. Besonders bei Wasserversorgern mit privater Mehrheitsbeteiligung findet diese Methodik kaum Resonanz (Haug 2006, S. 44 f.). Der Grad der Vollständigkeit der Antworten ist ein weiteres Problem, das sich bei dieser Methodik zur Datenerhebung stellt, da es dabei eine sehr große Bandbreite gibt. (Kletzan/ Url 2003, S. 394; Haug 2006, S. 44; Kirkpatrick/Parker/Zhang 2006 a, S. 148).

Eine andere Quelle sind öffentlich zugängliche Daten bzw. der Rückgriff auf bereits vorher gesammelte Datenpools (Zschille/Walter/von Hirschhausen 2010, S. 207). Da hier aber nur hoch aggregierte Daten enthalten sind, können verzerrende Einflüsse nicht eliminiert werden. So kritisieren Oelmann u. a. (2009, S. 842), dass weder die Annahme, dass die Umsätze stets kostendeckend sind noch die Nicht-Berücksichtigung der unterschiedlichen Höhen der Konzessionsabgaben, der Wasserentnahmeentgelte und der Ausgleichszahlungen an die Landwirtschaft zu brauchbaren Ergebnissen führen. Andere Probleme, die bei einer solchen Datenbasis auftreten, hängen mit der Tatsache zusammen, dass die Daten nicht explizit für diesen Zweck erhoben wurden. Daher muss in Kauf genommen werden, dass einige Variablen nicht in dem Sinne definiert wurden, wie sie für einen Effizienzvergleich benötigt werden (Coelli/Walding 2005, S. 17) oder gar gänzlich fehlen (Estache/Kouassi 2002, S. 142).

Die beste Datengrundlage besitzen die Studien, die auf die Daten einer Regulierungsbehörde zurückgreifen konnten. Hier sind die Unternehmen verpflichtet, ihre Daten an die Behörde in der vorgegebenen Qualität zu liefern. 


\section{Input- bzw. Outputgrößen und Kostentreiber}

Bei der Wahl der Inputgrößen ist zwischen monetären und physikalischen Größen zu unterscheiden, während bei den Outputs stets physikalische Größen verwendet werden.

Für die Wahl von monetären Inputs spricht, dass diese leichter zu erheben sind. Dabei ist zu entscheiden, wie aggregiert die Daten einfließen sollen. Gegen eine zu tiefe Aufschlüsselung spricht, dass bei der DEA mit der Zunahme an Inputvariablen die Anzahl der als effizient gewerteten Unternehmen zunimmt und somit die Diskiminierungskraft der Methode abnimmt (Tupper/ Resende 2004, S. 32). Außerdem stellt sich das Problem der willkürlichen Abgrenzung (Coelli/ Walding 2005, S. 18), so dass die Einbeziehung der Gesamtkosten oder deren Aufspaltung in Kapitalkosten und laufende Kosten zu bevorzugen ist. Von den laufenden Kosten sind dabei jedoch noch die Positionen Konzessionsabgabe, Wasserentnahmeentgelt und Ausgleichszahlungen an die Landwirtschaft abzuziehen. Diese drei Positionen unterscheiden sich je nach Stadt bzw. Bundesland teilweise deutlich voneinander, ohne dass das Versorgungsunternehmen darauf Einfluss nehmen kann.

Eine weitere entscheidende Frage ist, wie die Kapitalkosten in Anrechnung gebracht werden. Da diese bei einer Versorgungsaufgabe wie der Wasserversorgung einen Großteil der Kosten ausmachen, ist deren adäquate Berücksichtigung entscheidend für den Erfolg des Effizienzvergleiches. Außerdem weisen die einzelnen Wassernetze eine unterschiedliche Altersstruktur und abweichende Nutzungsdauern auf, so dass der Vergleich der Kapitalkosten schwierig ist. In einigen Studien wird diese Kostenposition durch eine physikalische Größe wie die Länge des Versorgungsnetzes approximiert. Dabei werden jedoch nicht alle Kapitalanlagen, wie beispielsweise Pumpen, miteinbezogen und auch Abschreibungen auf die Kapitalanlagen werden nicht berücksichtigt (Aubert/Reynaud 2005, S. 393).

Alternativ kann auch das bei der Anreizregulierung Einsatz findende Verfahren zur Standardisierung der Kapitalkosten analog angewandt werden. Die Standardisierung soll die Vergleichbarkeit der Kapitalkosten trotz unterschiedlicher Altersstruktur der Anlagen, Abschreibungs- und Aktivierungspraktiken ermöglichen. Um eine solche Verrechnung auch in der Wasserwirtschaft durchführen zu können, müssen die Kostenpositionen, die unter den Kapitalkosten zusammengefasst werden sollen, zunächst festgelegt werden. Als Anhaltspunkte könnten dabei die Bestimmungen in $\S 5$ Abs. 2 und $\S \S 6$ und 7 der Stromnetzentgeltverordnung (StromNEV) bzw. Gasnetzentgeltverordnung (GasNEV) dienen, auf die in $\S 14$ Anreizregulierungsverordnung (ARegV) verwiesen wird. Dort finden sich Aussagen über die anzusetzende Höhe der Fremdkapitalkosten ( 5 Abs. 2), über die kalkulatorischen Abschreibungen ( $(6)$ und zur kalkulatorischen Eigenkapitalverzinsung (§7). Um bei diesen Positionen eine Vergleichbarkeit zu erreichen, wird eine einheitliche Nutzungsdauer für jede Anlagegruppe ermittelt. Diese sind in Anlage 1 StromNEV bzw. Anlage 1 GasNEV festgeschrieben. Eine vergleichbare Aufstellung existiert für WVU nicht, wäre jedoch für eine faire Berücksichtigung der Kapitalkosten in einem Effizienzvergleich eine verlässliche Grundlage. Weiterhin muss eine gerechtfertigte Eigenkapitalverzinsung, die auch die netzspezifischen Wagnisse abdeckt, bestimmt werden.

Da dieses Vorgehen mit einem sehr hohen Aufwand verbunden ist und somit bei einem alleinstehenden Effizienzvergleich den Umfang sprengt, stünden nur bei einer gleichzeitigen Regulierung durch eine staatliche Behörde Aufwand und Nutzen in einem angemessenen Verhältnis. 
Andernfalls kann auch eines der oben beschriebenen Verfahren zur Behandlung der Kapitalkosten angewandt werden, wobei man sich dann deren Schwächen bewusst sein sollte.

Der Vorteil von physikalischen Inputs ist, dass diese dem Gedanken der Bestimmung der technischen Effizienz am besten entsprechen (Picazo-Tadeo/Sáez-Fernández/González-Gómez 2009, S. 619). Die Information über den Produktionsprozess wird nicht durch unterschiedliche Preise, die die Unternehmen auf den Beschaffungsmärkten erzielen konnten, verfälscht. Um den komplexen Produktionsprozess in seiner Gesamtheit abzubilden, sollten jedoch zumindest teilweise auch monetäre Größen in die Berechnung einbezogen werden.

Die Versorgung der Bevölkerung mit Wasser ist in starkem Maße von den vorliegenden Umweltbedingungen abhängig. Somit variieren auch die Kosten mit den jeweiligen Strukturmerkmalen. Um zu überprüfen, welche einen signifikanten Einfluss auf die Kosten haben, um diese dann im Effizienzvergleich ausreichend berücksichtigen zu können, müssen vorab Regressionsund Korrelationsrechnungen durchgeführt werden. Bei den Korrelationsrechnungen wird ermittelt, inwieweit sich die endogenen Kosten durch die exogenen Umweltbedingungen erklären lassen. Es kann dabei auch untersucht werden, wie mehrere Kostentreiber zusammen auf die Kosten wirken, um ein Modell zu entwickeln, das einen möglichst hohen Erklärungsbeitrag der Kosten aufweist (Oelmann u. a. 2009, S. 842 f.).

In den Studien werden verschiedene Strukturparameter auf ihre Bedeutung für die Kosten untersucht. So stellen Oelmann u. a. (2009, S. 843 f.) fest, dass die Kombination der drei Variablen Verteilnetzlänge, Verteilnetz pro Hausanschluss und Metermengenwert (= Verteilnetzlänge zu entgeltlicher Wasserabgabe) über 70\% (Bestimmtheitsmaß $\left.\mathrm{R}^{2}=0,706\right)$ der Verteilkostenunterschiede der Gruppe der größten Versorger erklären. Auch konnten die erwarteten Vorzeichen beobachtet werden. Sowohl die Verteilnetzlänge als auch die Verteilnetzlänge pro Hausanschluss besitzen eine positive Korrelation mit den Kosten, wohingegen mit zunehmenden Metermengenwert die Kosten sinken, da dieser mit der Bevölkerungsdichte ansteigt. Daher ist diese Kombination nach Ansicht der Autoren gut geeignet, um eine Basis für einen Effizienzvergleich für diese Gruppe zu schaffen. Andere Parameter, um die Besonderheiten der Versorgungsaufgabe zu beschreiben, sind bspw.:

- Die Bevölkerungsdichte bzw. versorgte Bevölkerung pro km Netzlänge (Tupper/Resende 2004; Renzetti/Dupont 2008).

- Die Bodenverhältnisse können bei ungünstigen Böden die Tiefbaukosten für den Leitungsbau um ca. 400\% von denen bei günstigen Böden abweichen lassen (Holländer u. a. 2009, S. 12).

- Die Topografie, da durch große Höhenunterschiede im Versorgungsgebiet insb. die Speicherung und Druckerhaltung einen großen Einfluss auf die Kosten hat. Zur Näherung für die spezielle Topografie kann die Differenz aus dem höchsten und dem niedrigsten Punkt im Versorgungsgebiet verwendet werden (Renzetti/Dupont 2008, S. 12; Zschille/Walter/von Hirschhausen 2010, S. 8).

- Auch die Herkunft des Wassers (Wasserquelle) wird häufig als Strukturparameter in die Analyse mit einbezogen, da sich bezüglich der Pump- und Aufbereitungskosten das Grund- deutlich vom Oberflächenwasser unterscheidet (Saal/Parker/Weyman-Jones 2007, S. 132). Durch die naturräumlichen Gegebenheiten ist die Auswahl an Alternativen beschränkt und auch der Transport über längere Distanzen ist zumeist unwirtschaftlich, so dass die Verwendung der Wasserquelle als exogen gegeben angenommen werden kann. Dieses legt eine Integration als 
Strukturparameter, beispielsweise als „Anteil an Grundwasser am gesamten Wasseraufkommen“(Zschille/Walter/von Hirschhausen 2010, S. 208) nahe, um Kosten, die hieraus entstehen, nicht dem Management anzulasten.

- Ebenfalls regelmäßig berücksichtigt werden die Wasserverluste, welche die Qualität der Leitungen wiedergeben. Sind sie z. B. auf Grund der Bodenbeschaffenheit unvermeidbar, können sie als exogen angenommen werden. Bedingt durch die abnehmende Wasserabgabe kann es vorkommen, dass die Wasserrohre aus hygienischen Gründen öfter durchgespült werden müssen. Auch diese Wassermengen werden unter die Wasserverluste gefasst. Falls die Wasserverluste mit unterbliebenen Instandsetzungen zusammenhängen, sind sie jedoch endogen durch das Management verursacht. Zwar ist grundsätzlich eine niedrigere Verlustrate vorteilhaft, da somit die nutzbare Wasserabgabemenge steigt, was einen positiven Effekt auf die Effizienz besitzt. Allerdings sind zur Vermeidung höherer Verlustraten hohe Instandhaltungskosten nötig, so dass die positiven Effekte auf die Effizienz aufgezehrt werden können (Haug 2007, S. 109). Grundsätzlich sollten exogene Wasserverluste als Strukturparameter in die Effizienzberechnung einfließen, endogene jedoch nicht.

- Daneben werden in den internationalen Studien noch weitere Strukturparameter verwendet, die jedoch aufgrund der speziellen Struktur für die deutsche Wasserwirtschaft kaum von Bedeutung sein dürften. Daher wird auf diese nicht vertiefend eingegangen.

Bei der Definition der Outputs stellt sich die Frage, durch welche Parameter die Leistung der WVUs am geeignetsten beschrieben werden kann. Viele der Studien verwenden die Parameter gelieferte Wassermenge und Anzahl Kunden bzw. Anzahl der Hausanschlüsse. García-Valiñas/ Muñiz (2007) benutzen zusätzlich die Länge des Verteilnetzes als Output, um die Größe des Versorgungsgebietes abzubilden. Lediglich Oelmann u. a. (2009, S. 842 f.) vertreten die Ansicht, dass weniger die Lieferung des Wassers die Hauptaufgabe der Versorger ist, sondern vielmehr die Vorhaltung von Kapazitäten für die Lieferung von Wasser. Daher beziehen sie auch Parameter wie Anzahl Pumpwerke oder Behälterkapazität in ihre Analyse mit ein. Da ein Großteil der anfallenden Kosten von der Größe des Netzes abhängt, diese jedoch nicht kurzfristig (z. B. bei einer abnehmenden Wasserabgabemenge) veränderbar sind, ist die ergänzende Betrachtung solcher Parameter zu empfehlen.

Je mehr Parameter in die Berechnung integriert werden, desto besser werden die Unterschiede in den jeweiligen Bedingungen der Unternehmen abgebildet. Es ist jedoch zu beachten, dass bei Hinzunahme von zusätzlichen Parametern die Modelle schwieriger zu berechnen sind und auch eine größere Anzahl von Unternehmen als effizient ausgewiesen wird.

\section{Ausreißeranalyse}

Bevor der eigentliche Effizienzvergleich durchgeführt werden kann, muss überprüft werden, ob in den Unternehmensdaten Ausreißer enthalten sind. Ausreißer, die auf Grund von Alleinstellungsmerkmalen oder Datenfehlern eine besonders hohe Effizienz aufweisen, können die Effizienzgrenze und damit auch die Effizienzwerte vieler oder gar aller Unternehmen beeinflussen. Die Entscheidung zwischen Ausreißern und effizienten Unternehmen, deren Besonderheiten nicht ausreichend berücksichtigt wurden, ist schwierig und sollte nicht rein automatisiert erfolgen. 
Zschille/Walter/von Hirschhausen (2010) verwenden den Supereffizienzansatz, in dem sie jedes Unternehmen mit allen anderen vergleichen (Banker/Gifford 1998). Es können somit Unternehmen einen Effizienzwert größer als 1 erreichen. Ab einem Wert von 1,2 gilt ein Unternehmen als supereffizient, so dass diese Unternehmen aus dem Datensatz entfernt werden. Außerdem wenden die Autoren noch die Kennzahl Umsatzerlöse pro Gesamtabgabemenge zur Identifikation von Ausreißern an. Ergeben sich hierbei für ein Unternehmen starke Abweichungen von der mittleren Spannweite, wird es gleichfalls aus dem Datensatz genommen. García-Sánchez (2006, S. 362) verwendet Input/Output-Relationen zur Ausreißeranalyse. Weicht dabei ein Unternehmen in mehreren Relationen oder in Relationen des gleichen Outputs zu verschiedenen Inputs zu weit vom Mittelwert ab, wird es als Ausreißer identifiziert und aus dem Datenpool eliminiert.

Bei besonders ineffizienten Unternehmen kann ein Mindesteffizienzwert vergeben werden. Dieser liegt in der deutschen Anreizregulierung bei 60\% (§ 12 Abs. 4 ARegV) und könnte für WVU ähnlich hoch angesetzt werden. WVU, die aufgrund mangelhafter Daten nicht in der Berechnung berücksichtigt werden konnten, sollten ebenfalls diesen Mindesteffizienzwert erhalten.

\section{Der Effizienzvergleich}

Zum eigentlichen Effizienzvergleich stehen die beiden Methoden DEA und SFA zur Verfügung. Idealerweise sollte der Effizienzvergleich pro Wertschöpfungsstufe erfolgen, was jedoch vielfach auf Grund der mangelnden Datenverfügbarkeit nicht möglich ist (Haug 2006, S. 47). Bei der Regulierung der Wasserversorgung in England und Wales verwendet OFWAT hingegen ein Modell, das die Effizienz in vier Teilbereichen (Wasserressourcen und Wasseraufbereitung, Wasserverteilung, Energiebedarf und Serviceleistungen) misst. Dabei werden für jeden Teilbereich eigene Kostentreiber definiert (Ofwat 2010, S. 16 f.).

\section{Auswahl der DEA-Spezifikation}

Die DEA findet in den internationalen Studien, die die Effizienz von Wasserversorgern messen, am häufigsten Anwendung. Es werden verschiedene Spezifikationen eingesetzt, um die jeweiligen Bedingungen adäquat zu beschreiben. Aus diesen Studien können Rückschlüsse für die deutsche Wasserversorgung gezogen werden.

\section{a) Input- vs. Outputorientierung}

Für die Ermittlung der Effizienzlinie ist die Orientierung nach Input oder Output irrelevant, da beide die selbe Effizienzlinie bestimmen. Unter der Annahme von konstanten Skalenerträgen liefern sogar die beiden Möglichkeiten exakt die selben Werte für alle Unternehmen. Dies ist bei variablen Skalenerträgen nicht gegeben, so dass hier die Wahl der Orientierung durchaus ins Gewicht fällt. 
In der Wasserwirtschaft wird in der Regel eine inputorientierte DEA eingesetzt. Es gibt aber auch Studien, die eine outputorientierte Version verwenden. Tupper/Resende (2004) liefern jedoch keine Begründung für ihre Wahl. Picazo-Tadeo/Sáez-Fernández/González-Gómez (2008, S. 36) verwenden diese Orientierung, da sie die Variable Wasserverluste als Merkmal für die Qualität des Wassernetzes als Output in ihre Berechnung einbeziehen.

Die Autoren, die eine Inputorientierung gewählt haben, begründen dies zumeist damit, dass das Management eines WVU größeren Einfluss auf die Inputs als auf die Outputs hat. Ein WVU versorgt ein bestimmtes geografisches Gebiet und somit sind die Outputs, also die abgegebene Menge bzw. die Anzahl der versorgten Bevölkerung, exogen gegeben (Coelli/Walding 2005, S. 14). Ein weiterer Vorteil ist, dass die ausgewiesenen Ergebnisse eine direkte Interpretation für mögliche Kosteneinsparungen liefern (Garci-Valinas/Muñiz 2007, S. 247). Somit kann eine zusätzliche Erkenntnis gewonnen werden, die eine wichtige Information für das Management darstellt.

Werden zusätzlich zu den Inputs und Outputs auch noch Strukturparameter in die Berechnung einbezogen, besitzt die Inputorientierung einen weiteren Vorteil. Es kann dann untersucht werden, inwieweit die einzelnen Strukturparameter auf die einzelnen Inputparameter wirken. Um hier Unterschiede entdecken zu können, werden inputorientierte Effizienzwerte benötigt (Picazo-Tadeo/González-Gómez/Sáez-Fernández 2009, S. 763).

Die Inputorientierung ist bei der Effizienzmessung mittels DEA in der internationalen Wasserwirtschaft Standard und somit auch für die deutsche Wasserwirtschaft zu empfehlen. Dies deckt sich auch mit der Effizienzmessung in anderen leitungsgebundenen Branchen wie der Strom- und Gasbranche.

\section{b) Skalenerträge}

Zur Berücksichtigung der Unternehmensgröße kommen für die deutsche Wasserwirtschaft die Annahme der konstanten (CRS), variablen (VRS) oder nicht-fallenden (NDRS) Skalenerträge in Frage. Nicht-steigende Skalenerträge spiegeln dagegen die Realität in der deutschen Wasserwirtschaft nicht passend wider, da in einem sehr stark fragmentierten Markt mit über 6.200 WVU der Großteil der Unternehmen unterhalb der optimalen Betriebsgröße, also unter steigenden Skalenerträgen, operieren dürfte.

Am weitesten verbreitet in der Literatur sind die variablen Skalenerträge. Konstante Skalenerträge werden in einer DEA-Methodik nur von García-Valiñas/Muñiz (2007) sowie Picazo-Tadeo/SáezFernández/González-Gómez (2008) verwendet, ohne dass eine Begründung für diese Wahl gegeben wird. Eine mögliche Begründung für den Einsatz dieser DEA-Spezifikation in der deutschen Wasserversorgung könnte in dem Wunsch einer Annäherung der vielen kleinen WVU an die optimale Betriebsgröße liegen. Da die unterschiedlichen Unternehmensgrößen und somit auch die Skaleneffekte nicht beachtet werden, werden Unternehmen, die die optimale Betriebsgröße nicht erreichen können, systematisch schlechter gestellt als unter der Annahme der variablen Skalenerträge. Es entstehen daher starke Anreize, sich der optimalen Betriebsgröße anzunähern. Da Unternehmen durch ihr festes Versorgungsgebiet nicht einfach die optimale Betriebsgröße erreichen können, werden in der Regel variable Skalenerträge unterstellt. Dem Management wird 
somit nicht angelastet, dass das Unternehmen nicht die optimale Betriebsgröße besitzt. Nur durch Kooperationen oder Fusionen können die kleinen Wasserversorger ihre Betriebsgröße beeinflussen. Die Versorgungsnetze bleiben allerdings auch hiervon unberührt, so dass Kosteneinsparpotentiale nur im Overhead erreicht werden könnten.

Kletzan/Url (2003) wenden dagegen sowohl eine CRS-DEA als auch eine VRS-DEA an. Sie vergleichen dabei die beiden resultierenden Effizienzwerte, um die Skaleneffizienz ermitteln zu können. Auch García-Sánchez (2006) sowie Coelli/Walding (2005) zerlegen mit Hilfe des VRSEffizienzwertes den CRS-Effizienzwert in die technische Effizienz und die Skaleneffizienz. Die hieraus gewonnene Zusatzinformation der Skaleneffizienz zeigt auf, inwieweit durch Anpassung der Unternehmen an die optimale Unternehmensgröße Kosteneinsparpotentiale ausgeschöpft werden können. Für eine mögliche Regulierungsbehörde ist diese Höhe ein guter Indikator für die Setzung zusätzlicher Anreize zur Unternehmenskonzentration. In den Studien konnten für die deutsche Wasserwirtschaft Skaleneffekte nur in kleinem Maße und auch nur für die Gruppe der kleinsten Wasserversorger nachgewiesen werden (Haug 2006, S. 47). Es sind dabei auch immer die jeweiligen örtlichen Rahmenbedingungen zu beachten, die vielfach eine Ausweitung der Versorgung unmöglich machen. In Großbritannien, wo im Zuge der Privatisierung 1989 ein starker Konzentrationsprozess begann, konnten bereits Größennachteile nachgewiesen werden (von Hirschhausen/Walter/Zschille 2009, S. 174).

Neben den konstanten und variablen Skalenerträgen kommen für die Wasserversorgung auch die nicht-fallenden Skalenerträge (NDRS) in Frage. Zwar wurde diese Form der DEA bisher noch nicht in einer Studie in der Wasserwirtschaft untersucht, doch aus dem Einsatz in der Anreizregulierung der deutschen Strom- und Gasbranche können Rückschlüsse auf einen möglichen Einsatz in der Wasserversorgung gezogen werden. Bei dieser Annahme werden den kleinen Wasserversorgern, die meist auf dem Land tätig sind, genau wie bei dem VRS-Ansatz Skalenineffizienzen zugestanden und sie können somit höhere Effizienzwerte erreichen als unter dem CRSAnsatz. Für sehr große Unternehmen gilt im Gegensatz zum VRS-Ansatz, das bei ihnen die fallenden Skalenerträge keine Berücksichtigung finden und ihnen daher unter Umständen niedrigere Werte zugewiesen werden.

Die Wahl der Skalenerträge für einen DEA-Effizienzvergleich in der deutschen Wasserversorgung hängt somit stark mit dem Ziel der Untersuchung zusammen. Sollen aus dem Effizienzvergleich starke Anreize zu Unternehmenszusammenschlüssen ausgehen, sollten CRS unterstellt werden. Für einen fairen Effizienzvergleich mit Berücksichtigung der durch die naturräumlichen Gegebenheiten vordefinierten Unternehmensgrößen sind die Annahmen der VRS bzw. NDRS zutreffender. Soll explizit das Kosteneinsparpotential durch Erreichung der optimalen Betriebsgröße angegeben werden, kann auch eine Kombination aus CRS und VRS angewandt werden.

\section{c) Einbezug der Kostentreiber}

Es können nicht beeinflussbare Umweltfaktoren auftreten, die eine sehr große Wirkung auf die Kosten und damit auf den berechneten Effizienzwert haben können. Das von Coelli u. a. (2006) vorgeschlagene Verfahren wird in Abb. 1 dargestellt. Zunächst wird die DEA mit den gängigen Inputs und Outputs durchgeführt, ohne die nicht-kontrollierbaren Einflüsse zu berücksichtigen 
(Brutto-Effizienzwert). Im zweiten Schritt werden die Brutto-Effizienzwerte auf die Umwelteinflüsse regrediert, um zu ermitteln, welche Einflüsse signifikante Auswirkungen auf die Effizienzwerte besitzen (Netto-Effizienzwert). Die Ergebnisse dieser Regression werden verwendet, um die Effizienzwerte anzupassen und von den nicht-beeinflussbaren Strukturvariablen zu bereinigen. Dazu werden die Inputniveaus aller WVUs an die schlechtesten Umweltbedingungen angepasst, um somit eine vergleichbare Grundlage für die Berechnung der Effizienzwerte zu schaffen (Zschille/Walter/von Hirschhausen 2010, S. 206). Dieses Verfahren wird in den meisten Studien angewandt, nur hinsichtlich des Regressionsverfahrens gibt es Unterschiede.

\begin{tabular}{|c|c|}
\hline $\begin{array}{l}\text { 1. Stufe: Brutto- } \\
\text { Effizienzmessung }\end{array}$ & $\begin{array}{c}\text { 2. Stufe: Regression und Netto- } \\
\text { Effizienzmessung }\end{array}$ \\
\hline $\begin{array}{l}\text { Je nach gewünschter Aussage: } \\
\text { CRS, VRS oder NDRS }\end{array}$ & $\begin{array}{l}\checkmark \\
\text { Regression auf Strukturparameter } \\
\text { (Tobit-, bootstrapped truncated } \\
\text { oder SFA-Regression }\end{array}$ \\
\hline Inputorientiert & $\begin{array}{l}\text { Anpassung der Inputniveaus mittels } \\
\text { Regressionsergebnisse }\end{array}$ \\
\hline $\begin{array}{l}\text { Keine Berücksichtigung der } \\
\text { Strukturparameter }\end{array}$ & Neuberechnung der Effizienzwerte \\
\hline Brutto-Effizienzwerte & Netto-Effizienzwerte \\
\hline
\end{tabular}

Abbildung 1: Zweistufiger Ansatz der DEA Effizienzmessung

Quelle: Eigene Darstellung

Kletzan/Url (2003) verwenden eine OLS-Regression, um den Einfluss der erklärenden Variablen auf den Effizienzwert zu untersuchen. Kritisch an dem Einsatz dieses Verfahrens ist, dass der Effizienzwert, der hier als abhängige Variable angenommen wird, nur Werte zwischen Null und Eins annimmt, wobei der Wert Eins häufig, nämlich bei allen effizienten Unternehmen, vorkommt. Durch den Störterm in der OLS-Regressionsgerade sind aber Werte zwischen minus und plus unendlich möglich, so dass dies nicht ausreichend die Realität widerspiegelt (Haug 2007, S. 108).

Um diese Kritik zu umgehen, verwenden andere Autoren eine bootstrapped truncated regression (Simar/Wilson 2007, S. 31 ff.). Bei dieser Methode werden Werte für die abhängige Variable nur in einem bestimmten Wertebereich verwendet, so dass Verzerrungen durch eine Wahrscheinlich- 
keitsmasse reduziert werden. Im Fall einer Effizienzmessung wird der Wertebereich auf das Intervall von Null bis Eins beschränkt, da der Effizienzwert ebenfalls auf dieses Intervall beschränkt ist und durch die Effizienzgrenze von Eins dort eine Wahrscheinlichkeitsmasse auftritt (Zschille/ Walter/von Hirschhausen 2010, S. 206). Dabei wird mit Hilfe einer Monte-Carlo Simulation, bei der durch vielfache Wiederholungen Pseudo-Datensätze auf Basis des vorhandenen Datenpools generiert werden, die Aussagekraft der geschätzten Koeffizienten erhöht (Renzetti/Dupont 2008, S. 5).

Neben dieser bootstrapped truncated regression wird vielfach eine Tobit Regression verwendet. Auch hierbei kann die abhängige Variable auf einen bestimmten Wertebereich begrenzt werden, in diesem Fall wieder auf das Intervall [0;1]. Ein Nachteil dieser zweistufigen Verfahren wird durch die Ergebnisse der ersten Stufe verursacht. Da die Effizienzen nicht direkt beobachtet werden können, sondern durch die DEA geschätzt werden müssen, können die in der zweiten Stufe eingesetzten abhängigen Variablen seriell korreliert sein, was zu unbrauchbaren Ergebnissen führen kann. Dies lässt sich aber durch ein bootstrapping vermeiden (Renzetti/Dupont 2008, S. 10).

\section{Auswahl der SFA-Spezifikation}

Neben der DEA wird die SFA in den Studien zum Effizienzvergleich in der Wasserwirtschaft vermehrt eingesetzt. Die SFA besitzt gegenüber der DEA den Vorteil, dass sie die Abweichungen von der Effizienzgrenze in Ineffizienzen und statistisches Rauschen unterteilen kann. Der Preis dafür ist, dass eine geeignete Produktions- bzw. Kostenfunktion gefunden werden muss, die sowohl die Daten möglichst passend abbilden kann als auch einfach genug sein muss, um eine gute Schätzung der Funktion zu erlauben.

\section{a) Mathematische Form der Kosten- bzw. Produktionsfunktion}

Auch bei der SFA stellt sich die Frage, ob eine inputorientierte Kosten- oder eine outputorientierte Produktionsfunktion gewählt werden soll. Ähnlich wie bei der DEA folgt der Großteil der Autoren der Inputorientierung, aber auch die outputorientierte Version fand schon in der Wasserwirtschaft Anwendung (Estache/Kouassi 2002, S. 11).

Für eine inputorientierte Kostenfunktion spricht neben den bereits bei der DEA angebrachten Argumenten, wie dem größeren Einfluss des Managements auf die Inputs, vor allem, dass bei einer Kostenfunktion mehrere Outputs berücksichtigt werden können (Lin 2005, S. 234). Da die Versorgungsaufgabe in der Wasserwirtschaft durch die Betrachtung eines einzelnen Outputs nur unzureichend abgebildet werden kann, ist die Einbeziehung von mehreren Outputs angebracht. Schwieriger als die Wahl zwischen Kosten- und Produktionsfunktion ist die Entscheidung über die mathematische Form der Funktion. Es lassen sich sowohl für die Cobb-Douglas- als auch für die Translog-Funktion Vor- und Nachteile finden. So führt Lin (2005) aus, dass bei seiner Untersuchung von 36 WVUs aus Peru eine Translog-Funktion nicht geeignet ist. Einerseits liegt eine hohe Multikollinearität vor - viele Korrelationen erreichen Werte von über 0,8 oder gar 0,9-, andererseits besitzt die Translog-Funktion für die kleine Grundgesamtheit an Unternehmen zu 
viele Freiheitsgrade. Es wären 44 Regressoren zu schätzen, was bei nur 36 Unternehmen nicht zu verlässlichen Ergebnissen führen würde. Daher wird eine Cobb-Douglas-Funktion verwendet (Lin 2005, S. 235). Estache/Rossi (2002, S. 143) führen ähnliche Argumente an, so dass auch sie auf die höhere Flexibilität der Translog-Funktion zu Gunsten der einfacheren Berechnung der CobbDouglas-Funktion verzichten.

Aubert/Reynaud (2005, S. 384) dagegen argumentieren, dass die Cobb-Douglas-Funktion zu unflexibel ist und somit den Produktionsprozess der WVUs nicht adäquat abbilden kann. Weiterhin können durch eine Translog-Funktion auch Skalen- und Dichteeffekte ermittelt werden, was bei einer Cobb-Douglas-Funktion nicht möglich ist. Vielmehr variieren hierbei die geschätzten Parameter für diese Größen nicht mit der Unternehmensgröße, sondern sind konstant für alle Unternehmen (Filippini/Zoric 2008, S. 173).

Neben dem Abwägen der jeweiligen Vor- und Nachteile besteht eine Möglichkeit die passende Funktionsform zu finden darin, die beiden Funktionsformen statistisch gegeneinander zu testen, wie es beispielsweise Kirkpatrick/Parker/Zhang machen (2006 b, S. 151). Sie verwenden dazu einen so genannten Likelihood-Quotienten-Test. Ihre Nullhypothese ist dabei die Verwendung der Cobb-Douglas-Funktion, die durch den Test nicht verworfen wird. Aubert/Reynaud (2005, S. 397) unterstützen ihre Wahl für die Translog-Funktion ebenfalls durch einen Test. Bei ihrem Chi-Quadrat-Test wird die Nullhypothese der Cobb-Douglas-Kostenfunktion bei einer Vertrauenswahrscheinlichkeit von $95 \%$ abgelehnt.

Neben diesen beiden Funktionsformen könnte auch die normierte lineare Funktion, wie sie in der Anreizregulierung verwendet wird, eine Möglichkeit in der Wasserversorgung darstellen. Als Normierungsgröße könnte beispielsweise die Anzahl der Hausanschlüsse dienen, die eine gute Approximation der Größe der Versorgungsaufgabe liefert. Diese Funktionsform bietet gegenüber der Cobb-Douglas-Funktion den Vorteil, dass die eingehenden Variablen nicht vorher logarithmiert werden müssen. Die Anzahl der zu schätzenden Parameter ist dabei identisch mit denen bei der Cobb-Douglas-Funktion und somit deutlich geringer als bei der Translog-Funktion.

Analog zur Anwendung der DEA stellt auch bei der SFA die Inputorientierung für WVUs das angebrachte Verfahren dar. Bezüglich der Funktionsform können sowohl die normierte lineare als auch die Cobb-Douglas oder die Translog-Funktion angewandt werden. Die Wahl hängt jedoch auch immer mit der Größe des Datensatzes zusammen. Auf Grund der hohen Anzahl an WVU in Deutschland sollten hierbei aber keine Restriktionen vorliegen, so dass die Wahl der flexibleren Translog-Funktion einige Vorzüge zu bieten hat.

\section{b) Verteilannahme der Effizienzterme}

Während die Verteilung der Störterme stets als Normalverteilung angenommen wird, ist die Verteilung der Ineffizienzen deutlich kritischer zu sehen. Grundsätzlich werden in der Literatur zur SFA die Halbnormalverteilung, die gestutzte Normalverteilung, die Exponentialverteilung und die Gammaverteilung genannt. In der Praxis werden aber vor allem die Halbnormal- und die gestutzte Normalverteilung verwendet.

Bei der Unterstellung einer Halbnormalverteilung wird durch die Festlegung des Mittelwerts $\mu$ auf 0 angenommen, dass sich der Großteil der Unternehmen nahe an der Effizienzgrenze befindet. 
Da dies bereits im Vorfeld eine Einschätzung der Ineffizienzen darstellt, wird eine gestutzte Normalverteilung einer unvoreingenommenen Effizienzbewertung gerechter. Für den Fall von $\mu=0$ fallen beide Annahmen zusammen (Estache/Rossi 2002, S. 140 f.).

Lin vergleicht in seiner Studie verschiedene Annahmen an die Verteilung der Ineffizienzen. Insgesamt kommen die Halbnormal-, die gestutzte Normal- und die Exponentialverteilung zum Einsatz. Es stellt sich heraus, dass die Ränge in den verschiedenen Modellen eine hohe Korrelation zueinander aufweisen, es jedoch bei einzelnen Unternehmen zu deutlichen Abweichungen kommen kann. So belegt ein Unternehmen beim halbnormalverteilten Modell den 21. Rang, beim exponentialverteilten Modell jedoch den 3. Rang (Lin 2005, S. 238). Ähnlich wie bei der Wahl der Funktionsform können auch bei der Wahl der unterstellten Verteilung statistische Tests zum Einsatz kommen. (Kirkpatrick/Parker/Zhang 2006 a, S. 151 f.).

Obwohl die Verteilung der Ineffizienzen keine große Auswirkung zu haben scheint, sollte auf Grund der höheren Flexibilität die Wahl auf die gestutzte Normalverteilung fallen. Hierbei wird nämlich nicht vorab ein Mittelwert festgelegt, sondern er bildet sich endogen aus dem Modell heraus und passt sich somit besser den Daten an als bei der Halbnormalverteilung, wo vorab der Mittelwert auf Null gesetzt wird.

\section{c) Berücksichtigung der Kostentreiber}

Um Einflüsse durch nicht-kontrollierbare Umweltfaktoren zu berücksichtigen, wurde von einigen Autoren ähnlich wie bei der DEA ein zweistufiger Ansatz verwendet. Zunächst wurden die Effizienzwerte ohne Berücksichtigung der Umweltfaktoren berechnet. Im zweiten Schritt wurden diese Ergebnisse dann auf die exogenen Variablen regrediert. Dabei tritt jedoch folgendes Problem auf: Um das Modell von Jondrow u. a. (1982) anwenden zu können, müssen die Ineffizienzen in der ersten Stufe als identisch verteilt angenommen werden. In der zweiten Stufe wird diese Annahme durch die Regression auf die Umweltfaktoren konterkariert, da durch die Regression unterstellt wird, dass ein funktionaler Zusammenhang zu den individuellen Gegebenheiten besteht und die Ineffizienzen eben nicht identisch verteilt sind (Kumbhakar/Lovell 2000, S. 264). Somit liefert dieses Vorgehen keine brauchbaren Ergebnisse.

Wie die Umwelteinflüsse dagegen adäquat in die SFA integriert werden können, hängt von der Annahme über ihre Auswirkungen ab. Es kann entweder unterstellt werden, dass sie sich auf die Form des Produktionsprozesses auswirken, oder aber direkt auf die jeweilige berechnete Effizienz des Unternehmens (Coelli/Perelman/Romano 1999, S. 251 ff.). Im ersten Fall wird für jedes Unternehmen eine eigene Produktionsfunktion angenommen, die von den jeweiligen Ausprägungen der Umweltbedingungen abhängt. Durch das Einbeziehen der Umweltbedingungen als Regressoren in die Produktionsfunktion erhält man Effizienzwerte, die von Einflüssen der Umweltbedingungen bereinigt sind.

Im zweiten Fall wird unterstellt, dass alle Unternehmen dieselbe Technologie, ausgedrückt durch eine einheitliche Produktionsfunktion, verwenden und sich die unterschiedlichen Umweltbedingungen auf die Abweichung von dem Best Practice Unternehmen auswirken. Hierfür wurden von mehreren Autoren (u. a. von Kumbhakar/Ghosh/McGuckin 1991, S. 279 ff.), Reifschneider/Stevenson (1991, S. 715 ff.) und Huang/Liu (1994, S. 171 ff.)) Modelle entwickelt, die eine explizite 
Funktion benutzen, um den Zusammenhang zwischen den Ineffizienzen und den firmen-spezifischen Faktoren abzubilden. Diese Modelle wurden von Battese/Coelli (1995, S. 325 ff.) auf Panel Daten erweitert. In diesem Modell werden die Ineffizienzen der Unternehmen als unabhängige, jedoch nicht identisch verteilte, nicht-negative Zufallsvariablen angenommen. Die Verteilungen der Ineffizienzen der einzelnen WVUs besitzen somit nicht mehr den gleichen Erwartungswert, da dieser von den firmenspezifischen Umweltfaktoren abhängt (Coelli u. a. 2006, S. 282 f.).

In den meisten Studien werden diese beiden Methoden verwendet. Lin testet in seiner Studie beide Verfahren und stellt heraus, dass seine Variablen zur Erfassung der Qualität besser als zusätzlicher Output in die Kostenfunktion integriert werden sollten, da andernfalls Unternehmen mit einer hohen Qualität benachteiligt würden (Lin 2005, S. 235).

Aubert/Reynaud (2005, S. 393) fügen sowohl einige Umweltbedingungen als Regressoren in die Kostenfunktion als auch einige als effizienzbeeinflussende Parameter in die Annahme des Erwartungswertes der Effizienzen ein. Sie unterscheiden also in ihrem Parametersatz zwischen solchen, die den Produktionsprozess beeinflussen und solchen, die Auswirkungen auf die berechnete Effizienz besitzen. Unter die erste Teilmenge fällt beispielsweise der Fremdbezug von Wasser, da bei dieser Form keine Aufbereitungsanlagen benötigt werden und es somit im Vergleich zu einem WVU, das nur Wasser aus Eigenförderung verwendet, $z u$ einem unterschiedlichen Produktionsprozess kommt. Dagegen wird der Form der Regulierung, unter der das Unternehmen in dem betrachteten Zeitintervall steht, nur ein Einfluss auf die berechnete Effizienz, nicht aber auf den Produktionsprozess zugeschrieben. Daher wird diese Variable bei der Ermittlung des Erwartungswertes berücksichtigt (Aubert/Reynaud 2005, S. 395).

Andere Autoren fügen die Umweltvariablen allesamt als Regressoren in die Kosten- bzw. Produktionsfunktion ein. Allerdings müssen um so mehr Parameter betrachtet werden, desto mehr Koeffizienten geschätzt werden. Daher können wieder Multikollinearitätsprobleme auftreten. Um diese Probleme zu umgehen, fügen einige Autoren die Umweltvariablen als Dummy-Variablen ein, falls sie eine hohe Korrelation zu anderen Parametern aufweisen. Es wird dabei jedoch in Kauf genommen, dass ein hoher Informationsgehalt verloren geht. So unterscheiden Filippini/ Hrovatin/Zoric (2008, S. 176) nur zwischen hohen und niedrigen Wasserverlusten, wobei der untere Quartilswert die Grenze bildet.

Bei der Berücksichtigung von Kostentreibern in der SFA sollte also unterschieden werden, wie sich diese auswirken. Daher muss für die zu beachtenden Parameter deutscher WVU jeweils eine Annahme über deren Auswirkungen getroffen werden. Während durch den Höhenunterschied im Versorgungsgebiet und die Art der Wasserquelle der Produktionsprozess durch Vorhalten mehrerer Druckstufen bzw. Aufbereitungsanlagen beeinflusst wird, wirken sich die Verteilnetzlänge, die Verteilnetzlänge pro Hausanschluss und der Metermengenwert vor allem auf die berechnete Effizienz des Versorgers aus. Durch ein längeres Verteilnetz oder einen höheren Metermengenwert wird sich der Produktionsprozess kaum verändern, die Kosten und somit auch die Effizienz hingegen schon. Dementsprechend sollten die ersten beiden Variablen als Regressoren und die letzten drei als effizienzbeeinflussende Parameter Berücksichtigung finden. 


\section{d) Panel oder Cross-Sectional Daten}

Ein großer Vorteil der SFA ist, dass problemlos Daten über einen längeren Zeitraum in die Analyse einbezogen werden können (Battese/Coelli 1992). Cross-Sectional Daten werden nur verwendet, wenn Beobachtungen nur für ein Jahr vorliegen, wie es bei Estache/Rossi der Fall ist (2002, S. 141). Dies dürfte aber besonders zu Beginn eines Effizienzvergleiches ein Problem darstellen, da nicht sofort Daten über mehrere Jahre vorliegen werden. Erst durch eine einheitliche Erfassung über einen längeren Zeitraum wird eine Analyse von Panel Daten überhaupt möglich. Solange muss mit Cross-Sectional Daten gearbeitet werden. Langfristig ist jedoch ein Vergleich von Panel Daten angebracht, um auch die Entwicklung über die Jahre abbilden zu können.

\section{Vergleich der Methoden}

Neben den bereits erwähnten Vor- und Nachteilen der Methoden ergeben sich weitere aus den besonderen Gegebenheiten in der (deutschen) Wasserwirtschaft, die sich besonders bei der Nutzung der SFA auswirken.

Da die Versorgungsaufgabe der einzelnen WVU stark von den naturräumlichen Gegebenheiten abhängt, ist nicht mit einem einheitlichen Produktionsprozess zu rechnen. So können ein Versorger einer Großstadt und ein Versorger eines ländlichen Gebiets nur sehr schwer miteinander verglichen werden. Daher ist es fast unmöglich, für einen ganzheitlichen Effizienzvergleich der WVU eine einheitliche Produktions- bzw. Kostenfunktion zu unterstellen, wie es bei der SFA nötig ist (Kletzan/Url 2003, S. 395). Um dieser Problematik auszuweichen, können die Unternehmen in Gruppen eingeteilt werden. Als Kriterium bietet sich dabei z. B. die Wasserabgabemenge an. So kann dann für jede Gruppe eine eigene Funktion vorgegeben werden, und es können auch für jede Gruppe individuelle Parameter in die Berechnung einbezogen werden (Oelmann u. a. 2009, S. 842 f.).

Es ist jedoch zu beachten, dass es durch die Einteilung in Gruppen zu Härtefällen kommen kann. Bei Unternehmen, die nahe an der Grenze zwischen zwei Gruppen liegen, kann eine Zuordnung $\mathrm{zu}$ jeweils unterschiedlichen Ergebnissen führen. Zudem erfolgt hierbei der Effizienzvergleich nur innerhalb der Gruppen, so dass ein globaler Effizienzvergleich unterbleibt. Zur Vermeidung dieser Schwachpunkte entwickelten Orea/Kumbhakar (2004, S. 169 ff.) den Ansatz der latent classes. Hierbei kann ebenfalls für jede Untergruppe eine eigene Funktion angenommen werden, die Unternehmen werden jedoch nicht fest in eine Gruppe eingeordnet, sondern sie gehören vielmehr mit einer bestimmten Wahrscheinlichkeit zu jeder Gruppe. Der Effizienzwert eines Unternehmens kann dann als mit den jeweiligen Wahrscheinlichkeiten gewichtete Summe der unter der Technologie der Gruppe ermittelten Effizienzen berechnet werden. Somit wird die Wahl der Zugehörigkeit der Unternehmen zu einer Klasse abgeschwächt und es erfolgt ein Vergleich mit allen Unternehmen, wobei jedoch auch berücksichtigt wird, dass nicht alle Unternehmen eine einheitliche Technologie verwenden.

Ein Vorteil der SFA ist es, dass sie Datenfehler durch den Fehlerterm abfangen kann, was bei der DEA nicht möglich ist. Bei deutschen WVU sollten Datenfehler im Vergleich zu anderen Branchen hingegen nicht von so großer Bedeutung sein. Daher fällt hier dieser Nachteil der DEA nicht 
so sehr ins Gewicht wie es etwa in Ländern mit weniger strengen Bilanzierungsrichtlinien der Fall ist. Durch die hohe Anzahl an Vergleichsunternehmen führen sowohl die DEA als auch die SFA auch bei einer hohen Anzahl von Parametern, die in der Berechnung berücksichtigt werden, zu verlässlichen Ergebnissen. Vor allem die DEA benötigt eine große Anzahl von Unternehmen, damit nicht ein Großteil der Unternehmen durch Alleinstellungsmerkmale als effizient ausgewiesen wird.

Insgesamt eignet sich somit insbesondere die DEA, da sich deren Schwächen durch die besonderen Bedingungen der deutschen WVU nicht so stark auswirken und somit die vielen Vorteile wie z. B. die Einfachheit zum Tragen kommen.

\section{Nach dem Effizienzvergleich}

Ist der eigentliche Effizienzvergleich abgeschlossen, müssen die Ergebnisse plausibilisiert werden. Anhand weiterer Berechnungen kann die Robustheit der Ergebnisse überprüft werden. Dafür bietet es sich bspw. an, mittels Sensitivitätsanalysen die getroffenen Annahmen oder verwendeten Strukturparameter auf ihre Relevanz zu überprüfen. Werden die Werte sowohl nach DEA als auch SFA berechnet, ist zu beachten, dass die Effizienzwerte nicht direkt miteinander verglichen werden können, da diese aufgrund der unterschiedlichen Methodiken andere Skalierungen aufweisen. Es sollte also lediglich ein Vergleich der Rangkorrelation der Ergebnisse (nach Spearman) erfolgen.

Bei der Interpretation ist zu berücksichtigen, dass Effizienzvergleiche nie einen punktgenauen Wert liefern, sondern dass es sich meist um ein Konfidenzintervall handelt. Somit sollten die Ergebnisse nicht direkt in die Erlösobergrenze übernommen werden. Es bietet sich daher an, analog zum Vorgehen der BNetzA in der Energiewirtschaft, anhand eines Best-of-Ansatzes sowohl eine SFA als auch eine DEA zu berechnen und dem WVU durch Bestabrechnung den jeweils maximalen Wert zuzuweisen. Dadurch wird die Gefahr einer Benachteiligung durch Modellierungsfehler verringert. Ein anderes Verfahren, welches bspw. Ofwat benutzt, ist die Einteilung in Effizienzklassen in 5\% Stufen. Anhand dieser Klasseneinteilung wird lediglich der „Catch-upEffekt" berechnet (Day 2010). Da in der deutschen Anreizregulierung der Best-of-Ansatz bereits angewendet wird, ist dieser gegenüber den Effizienzklassen vorzuziehen.

\section{Limitationen und Handlungsempfehlungen}

Bei Vorliegen eines kleinen Datensamples ist die Auswahl der Modellspezifikationen begrenzt. Bei der SFA ist mit einigen Restriktionen bei der Wahl des funktionalen Zusammenhangs zu rechnen, am besten ist deswegen die Cobb-Douglas-Funktion geeignet. Beim Einsatz der DEA können durch die wenigen Vergleichsunternehmen nicht so viele Inputs und Outputs in die Berechnung einbezogen werden. Andernfalls würde die Mehrheit der Unternehmen durch Alleinstellungsmerkmale ihr eigenes Benchmark setzen und fast alle Unternehmen würden als effizient ausgewiesen werden. Deshalb sollte der Vergleich nur anhand weniger aussagekräftiger Parame- 
ter erfolgen. Als Faustregel gilt, dass die Menge der Vergleichsunternehmen die Summe der Inputs und Outputs um mindestens das Zwei- bis Dreifache übertreffen sollte (Hammerschmidt 2006, S. 168). Insgesamt ist bei einem kleinen Datensample die DEA zu bevorzugen, da bei dieser Methode auch bei einer kleinen Datenmenge verlässliche Ergebnisse zu erwarten sind.

Problematisch ist der Fall von unvollständigen oder mangelhaften Daten. Der Verzicht einzelner Parameter, für die mangelhafte Daten vorliegen, ist insb. dann schwierig, wenn diese grundlegend für die Beschreibung der Leistungserbringung der Versorger sind. In diesen Fällen ist abzuwägen, ob der Verzicht auf das entsprechende Unternehmen sinnvoller ist. Der Einsatz der DEA ist problematisch, da sie keine Datenfehler abbilden kann. Somit wirken sich Fehler in den Daten direkt auf die Effizienzwerte der Unternehmen aus, was zu erheblichen Verfälschungen der Ergebnisse aller Unternehmen führen kann. Die SFA kann mit solchen Fehlern besser umgehen. Durch den symmetrischen Störterm werden diese Fehler abgefangen und wirken sich daher nicht im selben Maße auf die Effizienzwerte der Unternehmen aus.

Beim Vorliegen eines heterogen Datensamples, also wenn z. B. sowohl große als auch kleine Wasserversorger bzw. Direkt- und Fernversorger in dem Datensample enthalten sind, kann davon ausgegangen werden, dass kein einheitlicher Produktionsprozess vorliegt. Die Nutzung der SFA fällt aufgrund der fehlenden einheitlichen Funktion, die für alle Unternehmen die realen Bedingungen adäquat abbildet, somit schwer. Eine Klassenbildung, (Orea/Kumbhakar 2004, S. 169 ff.) ist bei einem heterogenen Datensample aus Direkt- und Fernversorgern wenig zweckmäßig, da es sich um disjunkte Gruppen handelt und keine Mischformen existieren. Es könnte ein Effizienzvergleich in den zwei Gruppen durchgeführt werden, wobei dann der Vergleich über die Gruppen ausbleibt. Da bei der DEA kein funktionaler Zusammenhang zwischen Inputs und Outputs vorgegeben werden muss, entfällt hier die Problematik. Es können ohne Weiteres alle Wasserversorger miteinander verglichen werden. Da dies allerdings nicht zielführend ist, sollte der Vergleich unter der Annahme von variablen Skalenerträgen durchgeführt werden. Um die unterschiedlichen Bedingungen berücksichtigen zu können, kann, wie bereits diskutiert, eine Regressionsrechnung angeschlossen werden. Somit ist die DEA für den Fall eines heterogenen Datensamples die bessere Wahl.

\section{Fazit}

Ausgehend von internationalen Studien und dem Einsatz in der Anreizregulierung in anderen Branchen wurden in diesem Artikel Rückschlüsse zur Durchführung einer Effizienzmessung in der deutschen Wasserwirtschaft gezogen. Dabei wurde festgestellt, dass vor der Effizienzmessung sowohl der Schaffung einer validen Datenbasis als auch der Identifikation der Kostentreiber eine zentrale Rolle zukommt.

Für den Effizienzvergleich muss zunächst die passende Methode ausgewählt werden. Dabei besitzt die DEA leichte Vorteile gegenüber der SFA. Ihre spezifischen Schwächen, wie die Nichtunterscheidung zwischen Ineffizienzen und Datenfehlern, fallen bei der Untersuchung deutscher WVU nicht so stark ins Gewicht wie die der SFA. Allerdings fällt hier besonders die notwendige Bestimmung einer einheitlichen Kostenfunktionsform schwer. 


\begin{abstract}
Andreas Hoffjan, Christian Lechtenberg and Lukas D. Schuchardt; How to Measure Efficiency in German Water Utilities? A Literature Survey of International Studies
\end{abstract}

DEA; SFA; structural parameter; cost driver; regulation

The German water supply industry shows large differences in the amount of the water prices. Only part of these can be explained by differential environmental factors. Other reasons are efficiency differences between the companies. To identify those, companies have to be compared with scientific efficiency analysis without disregarding the structural characteristics of the water supply. This article draws conclusions from international studies for measuring the performance in the German water supply industry.

\title{
Literaturverzeichnis
}

Aubert, Cécile und Arnaud Reynaud (2005), The Impact of Regulation on Cost Efficiency: An Empirical Analysis of Wisconsin Water Utilities, in: Journal of Productivity Analysis, 23 Jg., Heft 3, S. 383-409.

Banker, Rajiv D. und Jonathan L. Gifford (1998), Relative Efficiency Model for the Evaluation of Public Health Nurse Productivity, Mimeo.

Battese, George E. und Tim J. Coelli (1995), A model for technical inefficiency effects in a stochastic frontier production function for panel data, in: Empirical Economics, $20 \mathrm{Jg}$., Heft 2, S. 325-332.

Battese, George E. und Tim J. Coelli (1992), Frontier production functions, technical efficiency and panel data: With application to paddy farmers in India, in: Journal of Productivity Analysis, 3 Jg., Heft 1-2, S. 153-169.

Coelli, Tim J., Sergio Perelman und Elliot Romano (1999), Accounting for Environmental Influences in Stochastic Frontier Models: With Application to International Airlines, in: Journal of Productivity Analysis, $11 \mathrm{Jg}$., Heft 3, S. 251-273.

Coelli, Tim J., D. S. Prasada Rao, Christopher J. O'Donnell und George E. Battese (2006), An Introduction to Efficiency and Productivity Analysis, 2. Aufl. Berlin, Springer.

Coelli, Tim J. und Shannon Walding (2005), Performance Measurement in the Australian Water Supply Industry, In CEPA Working Papers Series, S. 1-37.

Day, Georg (2010), PR09/39: Relative efficiency assessment for operating expenditure 2008-09,

Dyckhoff, Harald und Katrin Allen (1999), Theoretische Begründung einer Effizienzanalyse mittels Data Envelopment Analysis (DEA), in: Zeitschrift für betriebswirtschaftliche Forschung, 51 Jg., Heft 5, S. 411-436.

Estache, Antonio und Eugene Kouassi (2002), Sector Organization, governance, and the Inefficiency of African Water Utilities Paper No. 2890, World Bank Policy Research Working.

Estache, Antonio und Martin A. Rossi (2002), How Different Is the Efficiency of Public and Private Water Companies in Asia?, in: The World Bank Economic Review, 16 Jg., Heft 1, S. 139-148.

Filippini, Nevenka Hrovatin und Jelena Zoric (2008), Cost efficiency of Slovenian water distribution utilities: an application of stochastic frontier methods, in: Journal of Productivity Analysis, 29 Jg., Heft 2, S. 169-182.

Garcia-Sanchz, Isabel M (2006), Efficiency Measurement in Spanish Local Government: The Case of Municipal Water Services, in: Review of Policy Research, 23 Jg., Heft 2, S. 355-372.

Garci-Valinas, Marie A und Manuel A. Muñiz (2007), Is DEA useful in the regulation of water utilities? A dynamic efficiency evaluation (a dynamic efficiency evaluation of water utilities), in: Applied Economics, $39 \mathrm{Jg}$., Heft 2, S. 245-252.

Hammerschmidt, Maik (2006), Effizienzanalyse im Marketing: Ein produktionstheoretisch fundierter Ansatz auf Basis von Frontier Functions, 1. Aufl. Gabler.

Haug, Peter (2007), Einfluss unterschiedlicher Organisationsstrukturen auf die Effizienz der Wasserwirtschaft - Das Beispiel Ostdeutschland, In Die Rolle der Kommunen in der Wasserwirtschaft - Hallesches Kolloquium zur Kommunalen Wirtschaft 2005, Schriften des IWH, Baden- Baden, Nomos Verlag, S. 91-114. 
Haug, Peter (2006), Ineffiziente Strukturen in der deutschen Kommunalwirtschaft? Das Beispiel der Trinkwasserversorgung in Ostdeutschland, in: Wirtschaft im Wandel, 2 Jg., Heft 12, S. 42-50.

von Hirschhausen, Christian (2009), Fallende Preise in der Wasserwirtschaft

- Hessen auf dem Vormarsch, in: DIW Wochenbericht, 10 Jg., S. 150-155.

von Hirschhausen, Christian, Matthias Walter und Michael Zschille (2009), Effizienzanalyse in der Wasserwirtschaft. Internationale Erfahrungen und Schlussfolgerungen für Deutschland, in: GWF Wasser/ Abwasser, Heft 2-3, S. $170-175$.

Holländer, Robert, Marcel Fälsch, Stefan Geyler und Sabine Lautenschläger (2009), Gutachten. Trinkwasserpreise in Deutschland - Wie lassen sich verschiedene Rahmenbedingungen für die Wasserversorgung anhand von Indikatoren abbilden?, Universität Leipzig.

Huang, Clief J. und Jin-Tan Liu (1994), Estimation of a non-neutral stochastic frontier production function, in: Journal of Productivity Analysis, $5 \mathrm{Jg}$., Heft 2, S. 171-180.

Jensen, Uwe, J Oberländer, C. Stiens und P. Wolffram (2008), Fairer Effizienzvergleich oder Milchmädchenrechnung?, in: Energiewirtschaftliche Tagesfragen, 58 Jg., Heft 7, S. 28-32.

Jondrow, James, C.A. Knox Lovell, Ivan S. Materov und Peter Schmidt (1982), On the Estimation of technical Inefficiency in the stochastic Frontier Production Function Model, in: Journal of Econometrics, 19 Jg., Heft 2-3, S. 233-238.

Kirkpatrick, Colin, David Parker und Yin-Fang Zhang (2006 a), An Empirical Analysis of State and Private-Sector Provision of Water Services in Africa, in: The World Bank Economic Review, 20 Jg., Heft 1, S. 143-163.

Kirkpatrick, Colin, David Parker und Yin-Fang Zhang (2006 b), An Empirical Analysis of State and Private-Sector Provision of Water Services in Africa, in: The World Bank Economic Review, 20 Jg., Heft 1, S. 143-163.

Kletzan, Daniela und Thomas Url (2003), Wirtschaftliche Kennzahlen und Effizienz in der österreichischen Siedlungswasserwirtschaft, in: WIFO-Monatsberichte, o. Jg., Heft 5, S. 389-403.

Kumbhakar, Subal C., Soumendra Ghosh und Thomas J. McGuckin (1991), A Generalized Production Function Approach for Estimation Determinants of Inefficiency, in: Journal of Business \& Economic Statistics, 9 Jg., Heft 3, S. 279-286.

Kumbhakar, Subal C. und C. A. Knox Lovell (2000), Stochastic Frontier Analysis, Cambridge University Press.

Lin, Chen (2005), Service quality and prospects for benchmarking: Evidence from the Peru water sector, in: Utilities Policy, 13 Jg., Heft 3, S. 230-239.

Oelmann, Mark (2005), Zur Neuausrichtung der Preis- und Qualitätsregulierung in der deutschen Wasserwirtschaft, 1. Aufl. Kölner Wissenschaftsverlag.

Oelmann, Mark, Christian Growitsch, Harald Kiesl und Hörg Schielein (2009), Vielfältige Chancen durch methodisch weiterentwickeltes Benchmarking, in: GWF Wasser/ Abwasser, Heft 150, S. 2-7.

Ofwat (2010), Relative efficiency assessments 2008-09 - supporting information, in: Ofwat.

Orea, Luis und Subal C. Kumbhakar (2004), Efficiency measurement using a latent class stochastic frontier model, in: Empirical Economics, 29 Jg., Heft 1, S. 169-183.

Picazo-Tadeo, Andrés, Francisco J. Sáez-Fernández und Francisco González-Gómez (2009), The role of environmental factors in water utilities' technical efficiency. Empirical evidence from Spanish companies, in: Applied Economics, 41 Jg., Heft 5, S. 615-628.

Picazo-Tadeo, Andrés J., Francisco González-Gómez und Francisco J. Sáez-Fernández (2009), Accounting for operating environments in measuring water utilities' managerial efficiency, in: The Service Industries Journal, 29 Jg., Heft 6, S. 761-773.

Picazo-Tadeo, Andrés J., Francisco J. Sáez-Fernández und Francisco González-Gómez (2008), Does service quality matter in measuring the performance of water utilities, in: Utilities Policy, 16 Jg., Heft 1, S. 30-38.

Reifschneider, David und Rodney Stevenson (1991), Systematic Departures from the Frontier: A Framework for the Analysis of Firm Inefficiency, in: International Economic Review, 32 Jg., Heft 3, S. 715-723.

Renzetti, Steven und Diane P. Dupont (2008), Measuring the Technical Efficiency of Municipal Water Suppliers: the Role of Environmental Factors, in: Department of Economics.

Saal, David S., David Parker und Tom Weyman-Jones (2007), Determining the contribution of technical change, efficiency change and scale change to productivity growth in the privatized English and Welsh water and sewerage industry: 1985-2000, in: Journal of Productivity Analysis, 28 Jg., Heft 1-2, S. 127-139.

Simar, Léopold und Paul W. Wilson (2007), Estimation and inference in two-stage, semi-parametric models of production processes, in: Journal of Econometrics, 136 Jg., Heft 1, S. 31-64.

Tupper, Henrique C. und Marcelo Resende (2004), Efficiency and regulatory issues in the Brazilian water and sewage sector: an empirical study, in: Utilities Policy, 12 Jg., Heft 1, S. 29-40.

Zschille, Michael, Matthias Walter und Christian von Hirschhausen (2010), Ineffizienz und Strukturunterschiede in der deutschen Wasserversorgung, in: Zeitschrift für öffentliche und gemeinwirtschaftliche Unternehmen, 33. Jg., Heft 3, S. 201-216. 
Methoden zur Effizienzmessung in der deutschen Wasserversorgung

\section{Gesetzesverzeichnis}

Anreizregulierungsverordnung (ARegV) in der Fassung der Bekanntmachung vom 29.10.2007 (BGB1. I S. 2529)

Bundesgerichtshof (BGH) vom 2.2.2010, KVR 66/08, OLG Frankfurt am Main - 11 W 23/07

Energiewirtschaftsgesetz (EnWG) vom 7.7.2005 (BGB1. I S. 1970, 3621) in der Fassung vom 21.8.2009 (BGB1. I S. 2870)

Gasnetzentgeltverordnung (GasNEV) vom 25.7.2005 (BGB1. I S. 2197) in der Fassung vom 3.9.2010 (BGB1. I S. 1261)

Stromnetzentgeltverordnung (StromNEV) vom 25.7.2005 (BGB1. I S. 2225) in der Fassung vom 3.9.2010 (BGB1. I S. 1261) 